\title{
Obesity Management
}

\section{Economic impact of childhood obesity on health systems: a systematic review}

\author{
F. Pelone, M. L. Specchia, M. A. Veneziano, S. Capizzi, S. Bucci, A. Mancuso, W. Ricciardi and \\ A. G. de Belvis
}

Institute of Hygiene, Catholic University of the Sacred Heart, Rome, Italy

Received 14 July 2011; revised 8 November 2011; accepted 8 November 2011

Address for correspondence: Antonio Giulio de Belvis, Institute of Hygiene, Catholic

University of the Sacred Heart, Largo

Francesco Vito, 100168 Rome, Italy.

E-mail: debelvis@rm.unicatt.it

\begin{abstract}
Summary
The primary purpose of this study is to analyse the costs related to childhood obesity $(\mathrm{CO})$ with reference to different models of healthcare systems. A systematic review of the economic impact of $\mathrm{CO}$ on healthcare systems was conducted by searching the main electronic scientific databases. Cost-of-illness (COI) analyses of children aged under 18 years who had been diagnosed as overweight or obese published up to July 2010 were considered. Short- and long-term consequences of $\mathrm{CO}$ were taken into account. In order to appraise the quality of the included studies, the British Medical Journal referees' checklist was used. About 3,844 COI analyses were initially found and 10 were finally considered in the current review: two studies referred to Beveridge and eight referred to Voluntary health insurance models. No studies have been conducted within a Bismarck model. Six studies considered in-patient costs, four studies estimated outpatient and primary care costs and seven studies considered pharmaceutical costs. The average quality of the included analyses was medium. The analysis confirmed the significance of $\mathrm{CO}$ related costs and the heterogeneity among available studies, which made it impossible to compare the different healthcare models.
\end{abstract}

Keywords: Childhood obesity, economics, systematic review.

obesity reviews (2012) 13, 431-440

\section{Introduction}

Childhood obesity $(\mathrm{CO})$ is one of the main public health challenges of the 21st century (1). Excess weight and obesity are among the most important public health issues in developed countries (2). Lifestyle factors such as sedentary behaviour and hypercaloric food consumption have led to dramatic increases in weight and body mass index (BMI) (3), and CO has become a worldwide epidemic that governments must face.

Current literature shows that being overweight or obese during childhood and adolescence increases the risk of developing several medical conditions (i.e. diabetes mellitus, hypertension, cardiovascular diseases, respiratory ailments, depression, orthopaedic problems and main cancers) (4). Worldwide estimates currently identify 18 million overweight children (5). The number of overweight adolescents has tripled since 1980 and the prevalence of obesity in younger children has more than doubled (6). Approximately 10-30\% of European children aged 7-11 years and $25 \%$ of European adolescents are overweight or obese (7). Studies have shown that being overweight or obese in childhood and adolescence has adverse consequences such as premature mortality and physical morbidity in adulthood (8).

CO has been demonstrated to have important social and economic consequences (9), so it is necessary to assess its economic burden. The economic consequences of CO are typically categorized as direct, such as medical costs, and indirect, such as school/work absenteeism (10). In the USA, 
the direct costs of CO include annual drug prescriptions, emergency room visits and in-patient and outpatient costs. Costs related to outpatient care in the USA in 2002-2005 were $\$ 14.1$ billion (11) while in-patient costs were $\$ 237.6$ million (12). The estimated annual cost of treating obesityrelated illness in adults in the USA amounted to \$147 billion by 2008 (13). In almost all healthcare systems, above all in those providing universal coverage and guaranteeing the health status in the population, decisionmakers need, in an era of scarcity of resources and fiscal constraint, more evidence-based tools to be able to effectively allocate funds for prevention and therapy.

The primary purpose of this study is to analyse CO-related healthcare costs for different models of healthcare systems by investigating a possible relationship between their magnitude and alternative healthcare systems. A secondary purpose is to provide a quality appraisal of the reviewed studies.

\section{Methods}

A systematic review of the economic impact of $\mathrm{CO}$ on healthcare systems, according to methods provided by the Task Force on Community Preventive Services (TFCPS), was conducted (14). A literature search was performed using the following electronic databases: Cochrane Library, Database of Abstracts of Reviews of Effects, Cumulative Index to Nursing and Allied Health Literature, EconLit, Scopus, Health Technology Assessment Database, Medline, National Health Service Economic Evaluation Database (NHS EED) (http://www.crd.york.ac.uk/ CRDWeb/AboutNHSEED.asp), Health Business, Psyco Info and Google Scholar. Each search engine was scanned by means of a specific search string that combines a database-specific mix of subject headings and text words starting from the Medline search strategy (summarized in Box 1).

Furthermore, scientific articles were found using the Medline 'related articles' algorithm and reference lists of eligible studies.

The following inclusion criteria were used.

- Cost-of-illness (COI) analyses published up to July 2010 and written in English, German, French or Spanish were considered. COI analyses focused on children aged under 18 years with a diagnosis of being overweight or obese (BMI $\geq 85$ th percentile) were included (15).

- Incidence-based COI analyses that estimated lifetime costs of CO for newly diagnosed cases were considered, as well as prevalence-based analyses that included all medical and morbidity costs. Both short- and long-term CO-related consequences were taken into account.

- COI analyses reporting results of economic data for direct medical costs were considered.

\section{Box 1 Medline search strategy}

The following algorithm for a systematic search was developed:

((()((()((()(indirect cost*)) OR (*direct cost*)) OR (medical cost*)) OR (economic*)) OR (economic burden))) OR (payment)) OR (expen*))) OR (l(l(l(l'Cost-Benefit Analysis'[Mesh] OR 'Cost of Illness'[Mesh]) OR 'Healthcare Costs'[Mesh]) OR 'Cost Savings'[Mesh]) OR ('Costs and Cost Analysis'[Mesh] OR 'Hospital Costs'[Mesh] OR 'Cost Allocation'[Mesh])) OR 'Cost Control'[Mesh]) OR 'Delivery of Health Careleconomics'[Mesh]) OR 'Hospitalization/economics'[Mesh])) OR (()((indirect cost*)) OR (*direct cost*)) OR (medical cost*)) OR (economic")) OR (economic burden))))) AND ((l('Obesity'[Mesh] OR 'Overweight'[Mesh]) OR 'Body Mass Index'[Mesh])) OR (((fatness)) OR (overweight*)) OR (obes*)))) AND (((()('Adolescent'[Mesh] OR 'Child'[Mesh]) OR ('Infant'[Mesh] OR 'Infant Nutrition Disorders'[Mesh])) OR 'Child, Preschool'[Mesh])) OR ('Paediatric Nursing'[Mesh]))) OR ((()((()((young*)) OR (youth*)) OR (infan*)) OR (juvenil*)) OR (child*)) OR (teen*)) OR (nurs*)) OR $($ baby*))) OR (paediatric*)).

Assessment of the eligibility of the studies and data abstraction was independently conducted by four couples of reviewers (SB, AGdB; SC, MLS; FP, AM; WR, MAV). Each pair consisted of a health economist and a medical doctor.

Studies were selected according to the following criteria

- analysis of titles and abstracts;

- collection of the full text of potentially relevant studies;

- cross-linking of studies retrieved from different databases in order to find and remove duplicate articles;

- analysis of the full text in order to ensure that studies met eligibility criteria.

Data were extracted using a checklist adapted from the 'Economic Evaluation Abstraction Form' provided by the TFCPS (14).For each selected study, two teams of reviewers (SB, AGdB, AM, FP; MLS, SC, MAV, WR) independently assessed the methodological quality. The British Medical Journal referees' checklist developed by Drummond and Jefferson (16) was used in order to appraise the quality of the COI analyses included in the review. As only COI analyses were included in our review, items that characterized full economic evaluations (Table 1) were not included. To investigate the relationship between $\mathrm{CO}$ healthcare costs and alternative healthcare systems, each study was 


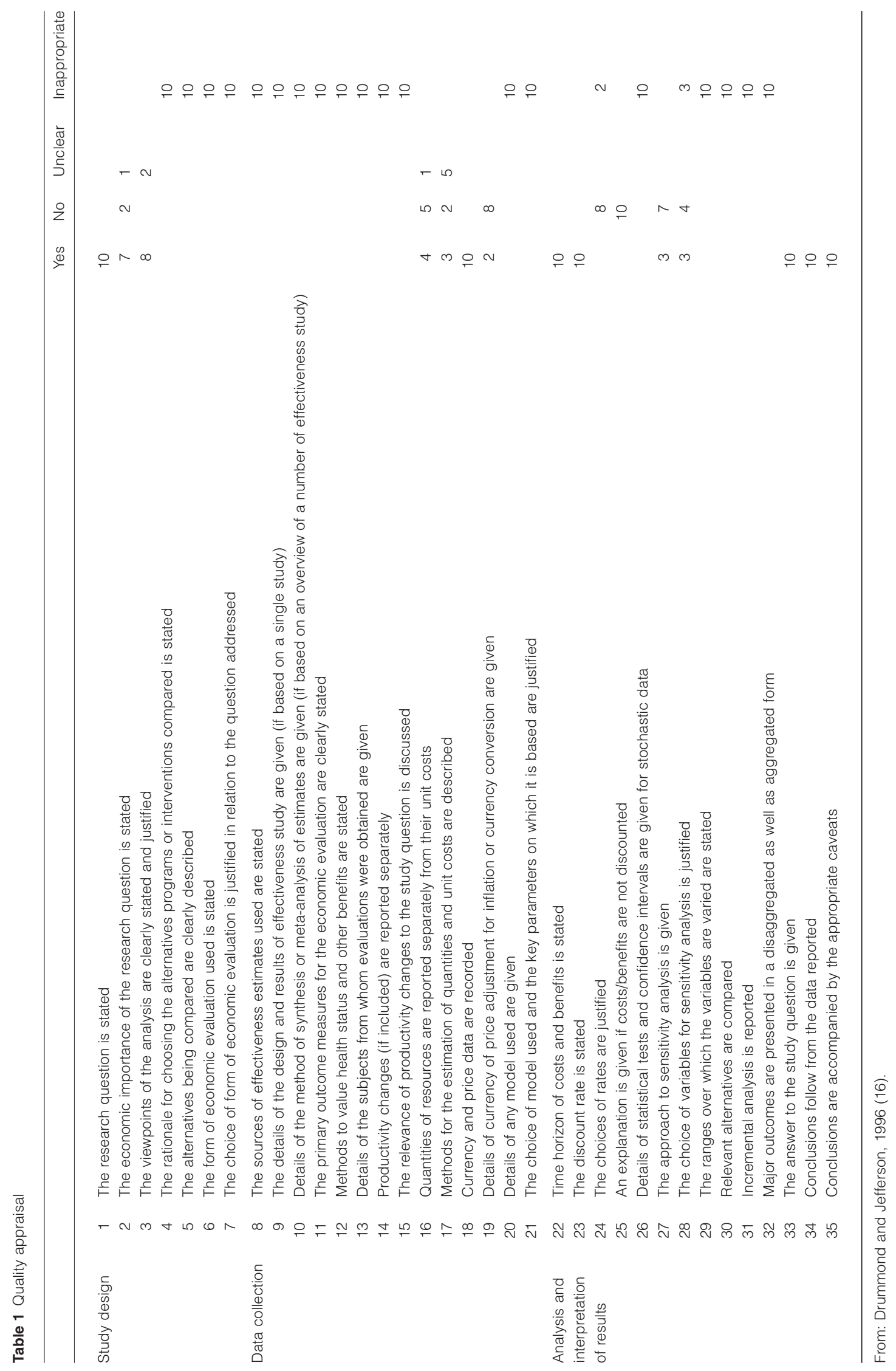


analysed according to healthcare components (e.g. hospital costs, physicians costs, drug prescriptions, primary and secondary care visits, and emergency and admission), methods (e.g. annual amount, amount trend and per-capita costs) and time frame.

\section{Results}

\section{Literature search results}

Figure 1 illustrates the literature search and selection process. The search strategy yielded 3,844 potentially relevant articles. After removing duplicates $(n=262), 3,381$ studies were excluded via title-screening because they did not focus on CO $(n=951)$ or economics $(n=2,430)$. By reviewing abstracts, 156 out of the 201 papers were excluded because they did not meet the inclusion criteria, the remaining 45 studies were included, 16 more studies were obtained by searching 'Related citations' on Pubmed and 14 studies were added after reviewing references, thus making a total of 75 articles.

From these 75 studies, 65 papers were rejected because they failed to meet inclusion criteria. At the end of the process, 10 COI analyses (1-10) were considered in the current review (Fig. 1). The features of the included studies are reported in Table 2.

\section{General overview}

Eight COI analyses were conducted in the USA $(11,12,17-$ 22), one in Canada (23) and one in Ireland (24). Most studies focused on the costs of CO by exploring the direct

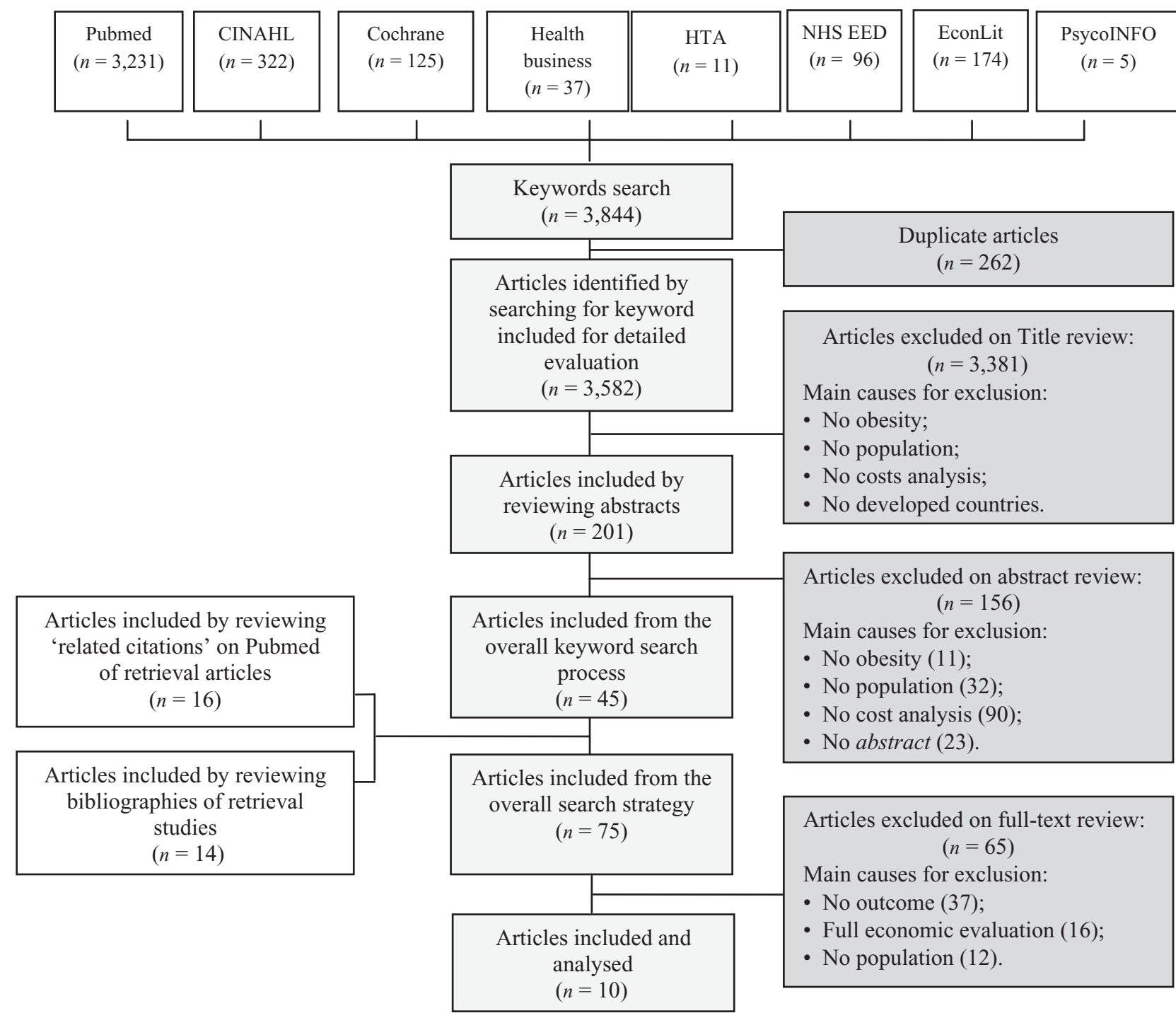

Figure 1 Study selection process. CINAHL, Cumulative Index to Nursing and Allied Health Literature; HTA, Health Technology Assessment Database; NHS EED, Economic Evaluation Database. 


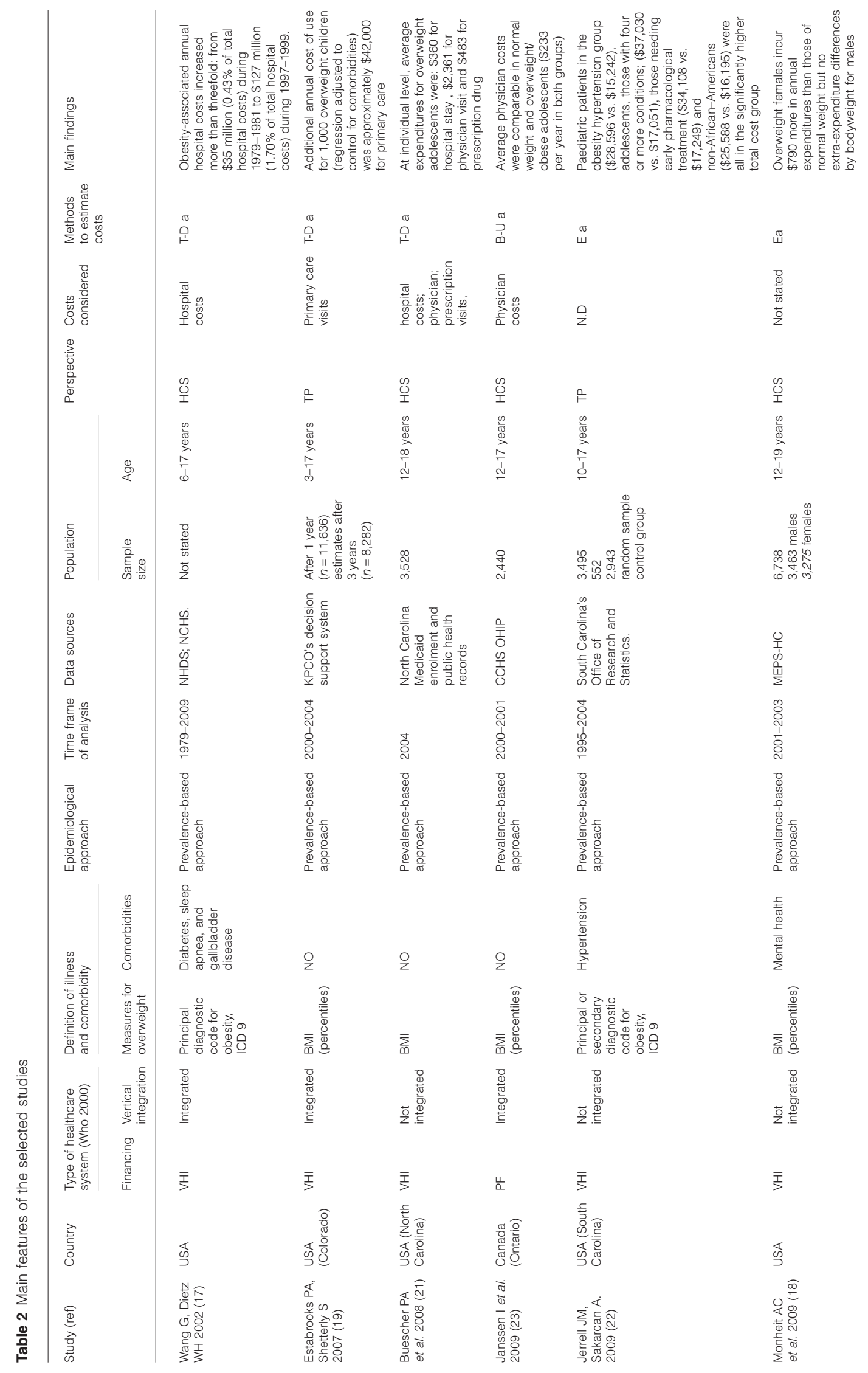




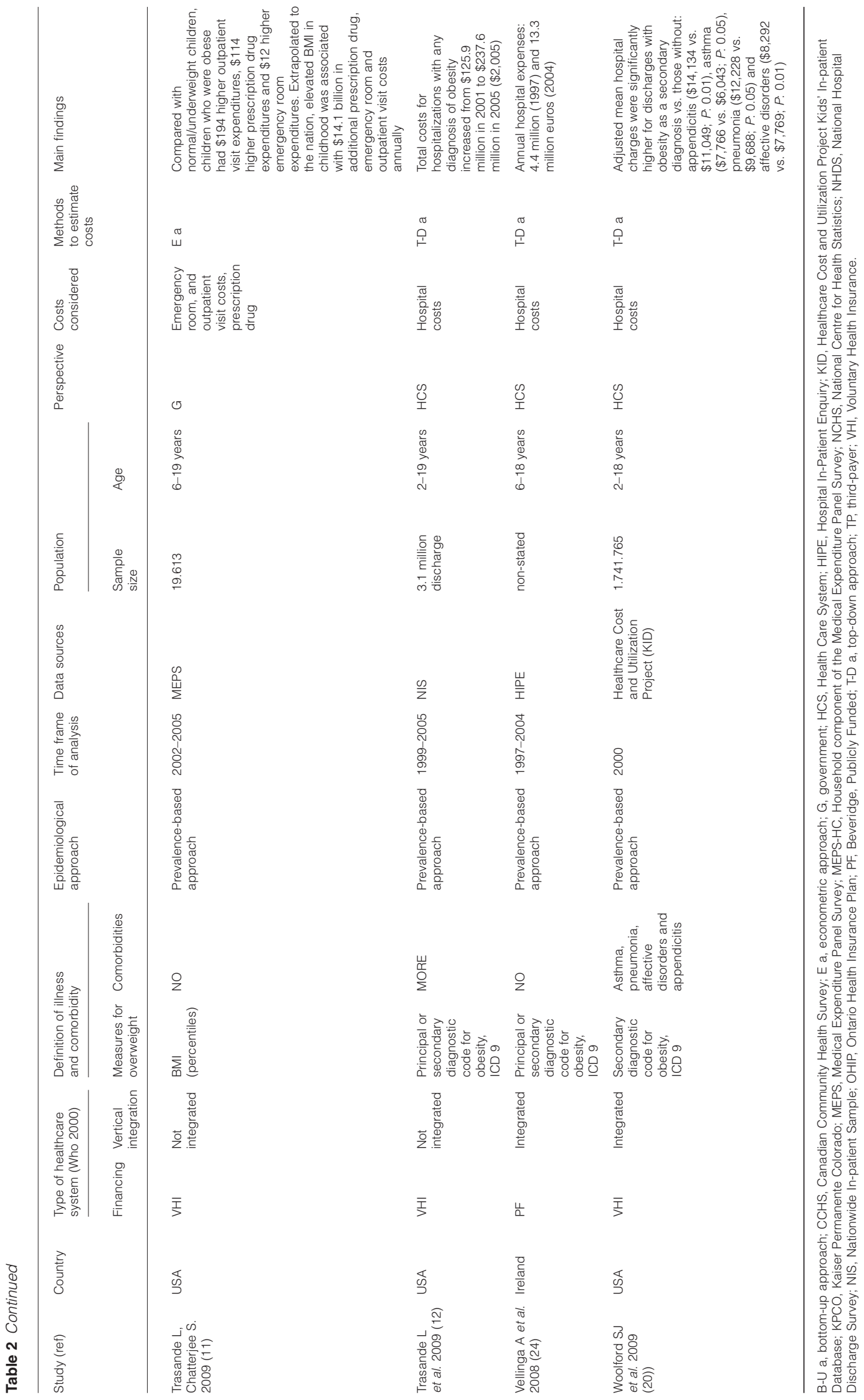


effects of obesity-related conditions such as hypertension (22), diabetes (17) and sleep apnea (17). Seven studies $(12,17,18,20,21,23,24)$ were conducted from the healthcare system perspective, considering only direct medical costs; two studies $(19,22)$ were conducted from the perspective of the third-party payer and only one analysis (11) considered the government point of view. All studies followed a prevalence-based approach, dealing with all medical and morbidity costs within the study period. None of the studies calculated CO costs by applying an incidencebased approach.

In six papers $(12,17,19-21,24)$, a 'top-down' approach was used to estimate the economic burden related to $\mathrm{CO}$ (i.e. hospital admissions and other disease-related costs). In three studies $(11,18,22)$, an econometric approach was used to calculate incremental costs between the cohorts of obese and non-obese children. Only one study (23) followed a bottom-up approach to estimate the costs by calculating the average outpatient costs of $\mathrm{CO}$.

Two of the COI studies $(23,24)$ were conducted within a 'Beveridge' healthcare system (free-of-charge health services with mainly public providers), eight studies $(11,12,17-22)$ within a 'voluntary health insurance' model (with mostly private, largely employer-based and out-ofpocket funding). None were conducted within a 'Bismarck' model (social assurance system with a statutory third-party payer).

Each included study was categorized according to the vertical integration of the healthcare system in which it was placed, in other words the arrangement whereby each healthcare system can offer, either directly or through others, a broad range of care and support services (Table 2).

Three studies $(19,23,24)$ estimated direct medical costs of $\mathrm{CO}$ in healthcare systems characterized by a high level of integration among revenue collection, financing, purchasing and provision function. Seven studies conducted in the USA $(11,12,17,18,20-22)$ estimated the economic impact of $\mathrm{CO}$ in healthcare systems belonging to a low level of vertical integration.

The economic burden in terms of direct medical costs was reported in each study. Six of the 10 studies considered in-patient costs $(11,12,21,24)$, four studies estimated outpatient and primary care costs $(11,19,21,23)$, and seven out of 10 studies considered pharmaceutical costs $(11,12,17,20-24)$. Allied healthcare costs were not included.

Unfortunately, it was not possible to compare the magnitude of CO healthcare costs according to different healthcare models, as the two studies that referred to the 'Beveridge model' applied different healthcare cost components (average physicians costs, 23), measures and time periods (total amount of annual hospital expenses in 1997 and in 2004, 24) than those of the eight studies referring to 'voluntary health insurance' models (11,12,17-22).

\section{Publicly funded health care}

Two studies considering the impact of CO on direct healthcare costs were targeted on publicly funded healthcare systems $(23,24)$. The COI from Ireland estimated the length of stay and hospital costs associated with obesity and related conditions for children between 6 and 17 years of age (24). One study from Ontario, Canada, estimated medical care costs in adolescents at the individual level (23).

Regarding in-patient costs, Vellinga et al. demonstrated that in Ireland the attributable length of stay (number of days in hospital for CO-related conditions per 1,000 d of hospital care) increased from 1.47 in 1997 to 4.16 in 2004. Furthermore, the annual hospital cost was calculated to be 4.4 million Euros in 1997, increasing to 13.3 million euros in 2004 (24).

The study conducted in Ontario by Janssen et al. found that the average physician costs were comparable in normal-weight and overweight/obese adolescents ( $\$ 233$ per year in both groups) (23).

\section{Privately funded health care}

All the studies based on privately funded health care came from the USA $(11,12,17-22)$.

Four of the eight studies specifically focused on hospitalization and in-patient physician costs due to $\mathrm{CO}$ $(12,17,20,21)$, two focused on outpatient and primary care costs $(11,19)$ and the remaining two estimated prescription drug expenditures associated with the care of CO $(18,22)$.

Two papers analysed outpatient costs. Trasande considered the following cost categories in 2002-2005: outpatient visits, emergency visits, office-based outpatient service, diagnostic and laboratory services, emergency visits, in-patient, and total number of in-patient days per episode. Trasande found that obese children had higher costs in the following categories: outpatient visits (\$194), prescription drugs (\$114) and emergency room visits (\$12). The results also highlighted the fact that the overall US annual cost of CO was $\$ 14.1$ billion and that obese children had 79 higher outpatient visit expenditures than healthy children (corresponding to $47 \%$ of the total cost for CO) (11). In the other outpatient COI, Estabrooks analysed the costs of extra visits in a sample of 1,000 overweight children, reported an annual overall cost of $\$ 42,148$ and $\$ 31,866$ for primary care sick visits and mental health visits, respectively (19).

\section{Quality appraisal}

All studies stated the research question within the study design (Table 1, item 1). In seven studies, the economic importance of the research was also stated (item 2) $(11,12,18-20,22,24)$. Four studies did not report resources and costs separately (item 16) $(11,19,20,22)$, and methods for the estimation of resource quantities and unit costs 
(item 17) were described in only three studies $(18,21,22)$. Currency and prices were recorded in all studies (item 18), but details of adjustment for inflation or currency conversion (item 19) were explained in only two studies $(11,12)$.

Time horizon of analyses was well described in all studies (item 22), and discount rates were stated (item 23); however, the choice of rates was not justified in any of the studies (item 24). No study gave an explanation if costs were not discounted (item 25). In addition, only three studies explained their approach to sensitivity analysis (item 27), thus justifying the choice of variables used (item $28)(18,20,22)$. In all studies, an answer to the study question was given (item 33), conclusions followed by the data were reported (item 34), and caveats were discussed (item 35). According to Drummond's checklist, the average quality of the analyses included in our review is medium because most studies do not give a real answer to all items.

\section{Discussion}

Obesity is of great importance from a health-policy perspective because of its prevalence in the population and the costs of treating the associated medical conditions in both the short and long term. Until recently, studies for children and adolescents were lacking, possibly due to the assumption that obesity-related health problems would occur only after longer periods of exposure and the latency effect. This view has been challenged by a number of studies, although not all findings were statistically significant and applicable to the whole child population (25).

As previous studies analysed the literature on COI related to $\mathrm{CO}$ (25), our review is the first to perform a systematic search and quality appraisal of literature and to investigate a possible relationship between magnitude of COI related to $\mathrm{CO}$ and healthcare models.

In spite of a starting number of 3,844 abstracts that fit the search strategy, only 10 were included into our review. We found only two studies reporting the burden of CO in national health systems. The lack of evidence confirms that in this priority area, more studies are required to properly inform and advise policy and allocate resources, in order to improve health and prevent illness.

Most studies (eight out of 10) were conducted in the USA, where research in health issues is likely to be more oriented to the burden of disease (26).

Our analysis points out the significance of CO healthcarerelated costs and emphasizes the need for political agendas to focus on early intervention, through health promotion and primary prevention of obesity. Unfortunately, the heterogeneity of the healthcare components, methods and timeframe analysed by the selected studies does not permit us to highlight possible relationships between the magnitude of CO-related healthcare costs and alternative healthcare systems. This issue confirms what has been stated on COI studies, where estimates of the healthcare costs of obesity vary depending on the method of calculation (27). For the same reason, it was not possible to find evidence of the differences between healthcare systems with regard to vertical integration. Our results point to a need for more comparable research, especially considering the fact that even the diseases that are included as being attributable to obesity differ in the available studies (27).

As for the quality appraisal of the included studies, we found out the following points.

Regarding the section 'Study design', most of the studies met the overall quality standards (i.e. statement and justification of the research question, of its economic relevance and of the analysis viewpoints). On the other hand, we found a lower quality level in the other two assessment section according to Drummond's: 'Data collection' and 'Analysis and interpretation of results'. For example, no study fully satisfied the following items: statement of the discount rate, justification of choices of rates and explanation of not discounting costs. Furthermore, just three studies stated the approach of sensitivity analysis, and gave the justification of variable selection.

This review is limited as a relatively small number of published COI exist in this field. It is also open to publication bias, as we have only included the published articles. Most of the research designs were unable to recognize with confidence that obesity is the underlying cause of higher costs. For example, in the cross-sectional studies and cohort analyses that relied on one measure of weight, it was possible that a third unobservable factor, such as an injury or mental health condition, might have caused both increased absences and increased weight (28). Estimates of COI could be influenced by the selection of obesity-related medical conditions as well as the double-counting of total costs of diseases that are complicated by comorbidities. No studies considered the long-term effects of CO, but concentrated on the short-term consequences, this is an area that requires future research.

'Costing' of an illness ought to be achieved through prevalence-based and incidence-based approaches. Prevalence-based COI studies do not quantify the longterm consequences of such chronic conditions as obesity in children. Therefore, an incidence-based COI assessment would be more appropriate (29). Nevertheless, we found no studies combining either approach, so to focus both on direct and indirect costs.

In pharmacoeconomic studies costs are classified into three categories: direct costs, indirect costs, intangible costs (27). However, none of the studies considered all three categories.

COI studies deal with diseases and not with interventions, as cost-effectiveness and cost-benefit studies do. So, they could demonstrate which diseases may require increased use of resources for prevention or treatment, but 
they encounter limitations in determining how to allocate resources and risk being overly heterogeneous in applied methods (30). Our analysis confirms such limitations.

COI analyses have been useful in some respects to conceptualize the magnitude of the CO epidemic, but do not represent a unique tool for successful guidance of policy in obesity-prevention. Nevertheless, COI studies would support the policymaking process by measuring the economic burden of a disease or diseases and estimating the maximum amount of money that could potentially be saved if a disease was prevented. Knowledge of the costs of an illness can help policymakers in deciding which diseases need to be addressed first by health care and prevention policies and how to allocate resources in the healthcare sector.

Based on the previous considerations:

1. there is an urgent need to intensify public health strategies, because of the growing prevalence of obesity and the related increasing economic burden. To achieve sustainable improvements in health and limit the growth of healthcare expenditures, decision-makers must recognize that positive lifestyles, behaviours, and social policy factors are the greatest factors in determining overall population health status (31).

2. Research in this field should be intensified, offering more comparable studies and studies of a wider range of health systems, in order to adequately inform decisionmakers and help them to formulate rational approaches to $\mathrm{CO}$.

3. Rigorous economic evaluations and reviews would play an important role in such a direction and should take into account the following issues:

- to consider the long-term effects of obesity in childhood - not only the short-term consequences;

- to use individual data instead of census data stored at national level in order to classify the 'exposure' (i.e. BMI, weight) and the 'effects' (prevalence/incidence of obesity-correlated diseases and costs, respectively), and to adjust for confounders through the adoption of regression models.

\section{Conflict of Interest Statement}

No conflict of interest was declared.

\section{Acknowledgement}

The methodological framework of our research has been presented at the EHMA Annual Conference on 'Managing Radical Change in Health: Quality, Efficiency, Equity' (30 June-2 July 2010, Lahti, Finland). The authors would thank Shaunna O'Brien and Caroline Canavan for the kind, final text revision.

\section{References}

1. World Health Organization. (2010). Childhood overweight and obesity on the rise. [WWW document]. URL http://www.who.int (accessed 20 December 2011).

2. Kelishadi R. Childhood overweight, obesity, and the metabolic syndrome in developing countries. Epidemiol Rev 2007; 29: 62-76.

3. Moayyedi P. The epidemiology of obesity and gastrointestinal and other diseases: an overview. Dig Dis Sci 2008; 53: 2293-2299. 4. Centers for Disease Control and Prevention. (2003). National Diabetes fact sheet: general information and national estimates on diabetes in the United States. [WWW document]. URL http:// www.cdc.gov (accessed 20 December 2011).

5. Ebbelung CB, Pawlak DB, Ludwig DD. Childhood obesity: public health crisis, common sense curve. Lancet 2002; 360: 473481.

6. Centers for Disease Control and Prevention. (2004). Overweight among U.S. Children and Adolescents. National Health and Nutrition Examination Survey. [WWW document]. URL http://www.cdc.gov (accessed 20 December 2011).

7. Lobstein T, Frelut M. Prevalence of overweight among children in Europe. Obes Rev 2003; 4: 195-200.

8. Reilly JJ, Kelly J. Long-term impact of overweight and obesity in childhood and adolescence on morbidity and premature mortality in adulthood: systematic review. Int J Obes 2011; 35: 891898.

9. Gortmaker SL, Must A, Perrin JM, Sobol AM, Dietz WH. Social and economic consequences of overweight among adolescents and young adults. N Engl J Med 1993; 329: 10081012 .

10. Cawley J. The economics of childhood obesity. Health Aff (Millwood) 2010; 29: 264-271.

11. Trasande L, Chatterjee S. The impact of obesity on health service utilization and costs in childhood. Obesity 2009; 17: 17491754.

12. Trasande L, Liu Y, Fryer G, Weitzman M. Effects of childhood obesity on hospital care and costs, 1999-2005. Health Aff (Millwood) 2009; 28: 751-760.

13. Finkelstein EA, Trogdon JG, Cohen JW, Dietz W. Annual medical spending attributable to obesity: payer- and servicespecific estimates. Health Aff (Millwood) 2009; 28: 822-831.

14. Task Force on Community Preventive Services. (2010). Community Guide Economic Evaluation abstraction form. [WWW document]. URL http://www.thecommunityguide.org/ about/EconAbstraction_v5.pdf (accessed 20 December 2011).

15. Kuczmarski RJ, Ogden CL, Grummer-Strawn LM et al. CDC Growth Charts: United States Advance Data from Vital and Health Statistics, No. 314. National Center for Health Statistics: Atlanta, GA, 2000.

16. Drummond MF, Jefferson TO. Guidelines for authors and peer reviewers of economic submissions to the BMJ. The BMJ Economic Evaluation Working Party. BMJ 1996; 313: 275-283.

17. Wang G, Dietz WH. Economic burden of obesity in youths aged 6 to 17 years: 1979-1999. Pediatrics 2002; 109: E81-E81. Erratum in: Pediatrics 2002; 109(6):1195.

18. Monheit AC, Vistnes JP, Rogowski JA. Overweight in adolescents: implications for health expenditures. Econ Hum Biol 2009; 7: $55-63$.

19. Estabrooks PA, Shetterly S. The prevalence and health care use of overweight children in an integrated health care system. Arch Pediatr Adolesc Med 2007; 161: 222-227.

20. Woolford SJ, Gebremariam A, Clark SJ, Davis MM. Incremental hospital charges associated with obesity as a secondary 
diagnosis in children. Obesity (Silver Spring) 2007; 15: 1895-1901. 21. Buescher PA, Whitmire JT, Plescia M. Relationship between body mass index and medical care expenditures for North Carolina adolescents enrolled in Medicaid in 2004. Prev Chronic Dis 2008; 5: A04.

22. Jerrell JM, Sakarcan A. Primary health care access, continuity, and cost among pediatric patients with obesity hypertension. $J$ Natl Med Assoc 2009; 101: 223-228.

23. Janssen I, Lam M, Katzmarzyk PT. Influence of overweight and obesity on physician costs in adolescents and adults in Ontario, Canada. Obes Rev 2009; 10: 51-57.

24. Vellinga A, O’Donovan D, De La Harpe D. Length of stay and associated costs of obesity related hospital admissions in Ireland. BMC Health Serv Res 2008; 8: 1-10.

25. John J, Wenig CM, Wolfenstetter SB. Recent economic findings on childhood obesity: cost-of-illness and cost-effectiveness of interventions. Curr Opin Clin Nutr Metab Care 2010; 13: 305-313.

26. Gross CP, Anderson GF, Powe NR. The relation between funding by the National Institutes of Health and the burden of disease. NEJM 1999; 340: 1914-1915.
27. Trogdon JG, Finkelstein EA, Hylands T, Dellea PS, KamalBahl SJ. Indirect costs of obesity: a review of the current literature. Obes Rev 2008; 9: 489-500.

28. Kortt MA, Langley PC, Cox ER. A review of cost-of-illness studies on obesity. Clin Ther 1998; 20: 772-779.

29. European Commission. (2005). The contribution of health to the economy in the European Union. Luxembourg: Office for Official Publications of the European Communities. [WWW document]. URL http://ec.europa.eu/health/archive/ph_overview/ documents/health_economy_en.pdf (accessed 20 December 2011) 30. Honeycutt AA, Segel JS, Hoerger TJ, Finkelstein EA. Comparing cost-of-illness estimates from alternative approaches: an application to diabetes. Health Serv Res 2009; 44: 303-320.

31. Docteur E, Oxley H (eds). OECD. Directorate for employment, labour and social affairs employment, labour and social affairs committee. (2003). Health-care systems: lessons from the reform experience. [WWW document]. URL http://www.lenus.ie/hse/bitstream/10147/44403/1/449.pdf (accessed 20 December 2011). 